\title{
Atitudes de usuários de um CAPSad frente às práticas de cuidado
}

\author{
Actitudes de los usuarios de CAPSad versus prácticas de cuidado
}

User Attitudes of a CAPSad Regarding Care Practices

\author{
Débora Mariana Stahelin \\ Centro Universitário Leonardo da Vinci (UNIVINCI), Guaramirim - SC/Brasil \\ ORCID: 0000-0002-6407-7686 \\ E-mail: deboramstahelin@hotmail.com
}

Jairson José Leichtweis Reis

Centro Universitário Leonardo da Vinci (UNIVINCI), Guaramirim - SC/Brasil ORCID: 0000-0002-9871-214X

E-mail: jairson7@gmail.com

Jean Paulo da Silva

Universidade Federal de Santa Catarina (UFSC), Florianópolis - SC/Brasil ORCID: 0000-0001-5173-1856

E-mail: jeanps.silva@gmail.com

Marieli Mezari Vitali

Universidade Federal de Santa Catarina (UFSC), Florianópolis - SC/Brasil ORCID: 0000-0003-0052-7788

E-mail: marielimezari@gmail.com

\begin{abstract}
Resumo
A presente pesquisa buscou investigar as atitudes de usuários de um CAPSad frente às práticas de cuidado. Caracterizada como levantamento de dados, de caráter exploratório e descritivo, com participação de 24 usuários. Foi aplicado um questionário composto por blocos de questões para caracterização sociodemográfica e frases afirmativas relacionadas ao contexto das práticas de cuidado no CAPSad, em formato de escala tipo Likert de cinco pontos. Os resultados apontam para um posicionamento favorável aos aspectos ligados às práticas de cuidado no CAPSad, sustentados por crenças consonantes com a política de redução de danos. Destaca-se que usuários que frequentavam oficinas terapêuticas apresentaram atitudes mais favoráveis às práticas de cuidado. O posicionamento predominantemente favorável ao atendimento individual como estratégia terapêutica enfatiza a necessidade de ampliar o escopo de ações coletivas. Refletese também que uma maior participação dos usuários nas ações do CAPSad possibilita maior participação na elaboração do plano terapêutico e em assembleias, sendo o controle social considerado uma estratégia de autonomia.
\end{abstract}

Palavras-chaves: Atitude; Serviços de saúde; Redução de danos.

\section{Resumen}

Esta investigación buscó investigar las actitudes de los usuarios de un CAPSad hacia las prácticas de cuidado. Caracterizado como recolección de datos, exploratorio y descriptivo, con la participación de 24 usuarios. Se aplicó un cuestionario compuesto por bloques de preguntas para la caracterización sociodemográfica y frases afirmativas relacionadas con el contexto de las prácticas asistenciales en CAPSad, en formato de escala Likert de cinco puntos. Los resultados apuntan a una posición favorable a los aspectos relacionados con las prácticas asistenciales en el CAPSad, sustentada en creencias alineadas con la política de reducción de daños. Es de destacar que los usuarios que asisten a talleres terapéuticos tienen actitudes más favorables a las prácticas de cuidado. El posicionamiento predominantemente favorable al cuidado individual como estrategia terapéutica enfatiza la necesidad de ampliar el alcance de las acciones colectivas. También se refleja que una mayor participación de los usuarios en la rutina y acciones de CAPSad permite una mayor participación en la elaboración del plan terapéutico y en las asambleas, considerándose el control social una estrategia de autonomía. 
Palabras clave: Actitud; Servicios de salud; Reducción de daños.

\begin{abstract}
This research aimed to investigate the attitudes of users of a CAPSad regarding care practices. Characterized as exploratory and descriptive data survey, with the participation of 24 users. A questionnaire consisting of blocks of questions for sociodemographic characterization and affirmative sentences related to the context of care practices in CAPSad, in a five-point Likert scale format, was applied. The results point to a favorable position on aspects related to care practices in
\end{abstract}

CAPSad, supported by beliefs in accordance with the harm reduction policy. It is noteworthy that users who attend therapeutic workshops have more favorable attitudes towards care practices. The predominantly favorable position for individual care as a therapeutic strategy emphasizes the need to broaden the scope of collective actions. It is also reflected that a greater participation of users in CAPSad routine and actions enables a greater participation in the elaboration of the therapeutic plan and in assemblies, and the social control exercised in the assembly can be considered a strategy of autonomy.

Keywords: Attitude; Health services; Harm reduction.

\section{Introdução}

Os problemas relacionados ao uso de drogas afetam as pessoas de diferentes maneiras, por diferentes razões e em diferentes circunstâncias e contextos. Existe uma tendência mundial que aponta para o consumo cada vez mais precoce de substâncias como álcool e outras drogas (Carlini et al., 2010), potencializando as situações de uso prejudicial, e tornando necessário o fortalecimento da rede de atenção à saúde em seus diversos níveis de complexidade, com ênfase na reabilitação psicossocial e reinserção, e considerando os cuidados centrados nas pessoas com problemas decorrentes do uso abusivo de álcool e outras drogas (Ministério da Saúde, 2015a; 2015b).

O que leva uma pessoa a ter envolvimento com o álcool e outras drogas, vai além da busca do efeito causado por estas substâncias. Diversos cenários devem ser considerados na tentativa de compreender esse fenômeno, tais como os contexto socioeconômico, político e cultural, disponibilidade de substâncias, o pensamento social e as ideias que circulam a respeito das drogas, as características da personalidade, a utilização de substâncias pelo grupo familiar, amigos ou outros grupos sociais importantes. Trata-se, portanto, de um problema multidimensional e global, que ultrapassa a simples relação entre um indivíduo e o consumo da substância psicoativa (Ferreira \& Luiz, 2004; Gonçalves \& Tavares, 2007).
A necessidade de definição de estratégias específicas para o enfrentamento dessa crescente problemática levou o Ministério da Saúde a criar, no âmbito do Sistema Único de Saúde, o Programa Nacional de Atenção Comunitária Integrada aos Usuários de Álcool e outras Drogas, via Portaria GM/336 de 30 de abril de 2002 (Portaria GM n. 336, 2002). Na rede de saúde do país, os CAPSad têm como desafio ser um dos principais mecanismos na consolidação das políticas de atenção ao usuário de drogas. Inseridos na rede de saúde pública, lhes garante uma intervenção cujo objetivo é a saúde integral do usuário e não somente a redução da demanda por drogas. $\mathrm{O}$ serviço considera ainda a necessidade de estruturação e fortalecimento de uma rede de assistência centrada na atenção comunitária, associada à rede de serviços de saúde e sociais, com ênfase na reabilitação e reinserção social dos usuários (Ministério da Saúde, 2015a).

A perspectiva teórica da psicologia social, com foco na cognição e percepção social, possibilita a compreensão de aspectos que envolvem as interações sociais dos indivíduos e grupos em variados contextos (Tróccoli, 2011). Assim, entende-se que por meio da investigação das atitudes e suas relações com crenças e normas sociais é possível buscar entendimento sobre a percepção e posicionamento dos usuários de um CAPSad frente às práticas de cuidado vivenciadas neste contexto de relação.

É por meio da percepção do meio social 
e dos outros que as pessoas organizam informações, as relacionam com afetos (positivos ou negativos) e desenvolvem uma predisposição para agir (atitude favorável ou desfavorável) em relação às pessoas, conceitos e aos objetos presentes no meio social. Assim, as atitudes possuem estreita relação com as noções de crença, valor e preconceito na medida em que apresentam elementos semelhantes em sua organização, e estes se instituem como agentes justificadores das atitudes (Lima \& Correia, 2013). Esse posicionamento atitudinal, na forma de uma avaliação frente a um objeto social, implica em um movimento ou predisposição à ação, podendo influenciar no engajamento dos indivíduos em comportamentos dentro de uma dinâmica social circunscrita em seu contexto, tendo sua manifestação demandada nos processos de interação social e pertenças grupais (Ajzen, 2005; Doise, 2001).

Com base no cenário atual, assume-se que o uso de álcool e outras drogas se tornou um tema preocupante para diversos setores da sociedade. As práticas de cuidado relacionadas a essas demandas, no contexto do CAPSad envolvem um modelo substitutivo ao padrão asilar, com objetivo de promover a vida, garantir o exercício da cidadania e a inclusão social, e envolve o Projeto Terapêutico Singular (PTS) específico para cada usuário. Dentre as possibilidades de práticas de cuidado identificadas pelo Ministério da Saúde (2015a) tem-se o acolhimento inicial, acolhimento diurno e noturno, atendimentos individuais, grupais, familiares e domiciliar, atenção à situações de crise, práticas corporais, expressivas e comunicativas, ações de reabilitação psicossocial e de redução de danos, entre outras práticas.

Dessa forma, buscar conhecer as atitudes dos usuários do CAPSad frente às práticas de cuidado se torna um empreendimento que pode resultar no desenvolvimento de estratégias que visem contemplar melhorias no acompanhamento dos usuários, tendo em vista que os resultados podem ser norteadores do trabalho das equipes de saúde do CAPSad e demais dispositivos da rede de atenção à saúde. Diante disso, a presente pesquisa teve por objetivo investigar as atitudes de usuários de um CAPSad frente às práticas de cuidado.

\section{Metodologia}

A presente pesquisa possui delineamento de levantamento de dados e caráter exploratório e descritivo. Participaram do estudo 24 usuários de um CAPSad. A amostragem se deu por conveniência. Os critérios de inclusão foram: usuários de ambos os sexos, com idade igual ou superior a 18 anos e que frequentavam o CAPSad há no mínimo três meses.

O instrumento para coleta de dados consistiu em um questionário estruturado, composto por um bloco com questões de caracterização sociodemográfica e um bloco com questões fechadas relacionadas ao contexto de participação do usuário no CAPSad. Além disso, para investigação das atitudes dos usuários frente às práticas de cuidado no CAPSad, utilizou-se um conjunto de frases afirmativas, como por exemplo: "Considero as assembleias de usuários importantes para a melhoria dos serviços do CAPSad", que eram respondidas com posicionamento favorável ou desfavorável em uma escala Likert variando em 1 - Discordo totalmente; 2 - Discordo em parte; 3 - Não concordo e nem discordo; 4 - Concordo em parte; e 5 - Concordo totalmente.

A aplicação do questionário foi individual e realizada no ambiente do CAPSad, em momentos em que o participante estivesse disponível, sendo iniciada após a assinatura do Termo de Consentimento Livre e Esclarecido. As questões foram lidas em voz alta pelo pesquisador, com o participante sentado ao seu lado para possibilitar a observação do formulário, especialmente na aplicação da escala. A resposta então era anotada no formulário pelo pesquisador.

As respostas foram tabuladas e os dados originados nas questões com resposta em escala de tipo Likert, as demais variáveis do 
instrumento e as de caracterização sociodemográfica foram analisadas utilizando estatística descritiva (medidas de tendência central e dispersão) e relacional (t-student e ANOVA), por meio do uso do software estatístico PSPP-GNU de distribuição livre.

O presente estudo respeitou as diretrizes e critérios estabelecidos na Resolução 466/12 do Conselho Nacional de Saúde (CNS) e o início da coleta de dados foi condicionado à aprovação do projeto pelo Comitê de Ética para Pesquisa com Seres Humanos sob protocolo CAAE: 57570516.9.0000.5358 e parecer de aprovação 1.646.251.

\section{Resultados}

A média de idade dos participantes foi de 49 anos, com mínimo de 26 e máximo de 68. Sendo 20 participantes do sexo masculino e 4 do sexo feminino. No que se refere à escolaridade, observou-se a prevalência do ensino fundamental como o maior nível de escolaridade. Ao investigar a principal fonte de renda, 6 participantes declararam não possuir uma fonte de renda e 7 declararam ter renda de no máximo um salário mínimo. Em relação ao estado civil dos participantes, 10 se declararam solteiros. Quanto ao contexto familiar, a maior parte $\quad(n=14)$ mora com o(a) esposo(a)/companheiro(a) e/ou outros familiares.

Ao serem questionados sobre a forma como acessaram o CAPSad, 7 procuraram por vontade própria, 7 por indicação de familiares, 7 por indicação de outros serviços de saúde, 1 por indicação do emprego, 1 por ordem judicial e 1 por indicação de amigos. Na questão referente ao tempo que o participante frequenta o serviço, do total $(\mathrm{n}=24), 16$ participantes frequentam a mais de um ano e 8 a menos de um ano. Sobre a frequência de participação desses usuários no CAPSad, do total $(n=24)$ a maioria $(n=19)$ frequenta o serviço de 1 a 2 dias por semana.

Quando questionados sobre como avaliavam a importância de ter um profissional de referência no CAPSad, quase a totalidade dos participantes $(\mathrm{n}=23)$ declararam ser importante. Além disso, a maior parte $(n=16)$ declarou que sempre consegue falar com o profissional de referência quando necessário. Em relação às oficinas realizadas no CAPSad, 17 participantes referiram participar das atividades desenvolvidas. Sobre os atendimentos individuais como parte do cuidado, a maioria dos participantes $(n=19)$ respondeu que realizam esse tipo de atendimento.

Questionados a respeito do uso de algum tipo de substância psicoativa nos últimos 15 dias, do total $(n=24)$ a maioria $(n=14)$ respondeu que fez uso. Dentre as substâncias utilizadas está o álcool como mais frequente (11), além de outras substâncias como cigarro/palheiro (5), maconha (4), crack (2) e cocaína (2), havendo uso de mais de uma substância por alguns usuários.

Quanto ao uso de medicamento psiquiátrico como terapêutica, 14 participantes afirmaram usar um ou mais tipos de medicamentos, enquanto 10 participantes referiram não usar nenhum medicamento. Neste sentido, constatou-se que dos 14 participantes que fazem uso de algum tipo de medicamento, as classes mais utilizadas foram a de medicamentos ansiolíticos $(n=7)$ e antipsicóticos $(\mathrm{n}=7)$.

As atitudes dos usuários foram investigadas a partir de frases afirmativas que buscavam seu posicionamento frente às práticas de cuidado e ações no CAPSad, obtendo-se escores em intervalo de 1 a 5 . Portanto, quanto maior o valor, maior a favorabilidade frente ao contexto da afirmativa apresentada. Ressalta-se que apesar de a amostra ser pequena, os dados analisados apresentaram distribuição normal, sendo possível assim empregar testes paramétricos para as análises.

Em relação aos aspectos ligados à perspectiva de Redução de Danos, os participantes foram questionados sobre seu posicionamento quanto a usar substâncias e frequentar o serviço de saúde, por meio das 
afirmações: "Penso que é certo a pessoa frequentar o CAPSad mesmo se continuar usando álcool ou outras drogas" obtendo-se $(\mathrm{M}=3,75 . \mathrm{DP}=1,54)$ e "Eu utilizo ou utilizaria o CAPSad mesmo usando álcool ou outras drogas" $\quad(\mathrm{M}=3,88 . \quad \mathrm{DP}=1,68) . \quad \mathrm{Em}$ complemento, ao comparar os escores obtidos analisando usuários que fizeram uso de substâncias psicoativas nos últimos 15 dias e os que não fizeram, verifica-se diferentes posicionamentos. Usuários que fizeram uso acreditam ser correto frequentar o CAPSad mesmo mantendo o uso, diferentemente dos usuários que não fizeram uso, que se posicionaram desfavoravelmente $[\mathrm{t}(22)=$ 5,436; $\mathrm{p}<0,01]$. Ressalta-se a relevância da diferença no que se refere ao direcionamento comportamental. Em que se verifica que tanto os usuários que fizeram uso quanto os que não fizeram referem comportamento ou intenção comportamental de acordo com sua crença. $\mathrm{Ou}$ seja, quem fez uso é mais favorável à possibilidade de o usuário frequentar o CAPSad mesmo tendo usado substância, e quem não fez uso refere que não frequentaria o CAPSad nessa condição $[\mathrm{t}(22)=4,705 ; \mathrm{p}<$ $0,01]$.

Outro fator que se relaciona ao contexto que sustenta a lógica de atuação baseada nas diretrizes do CAPSad é a continuidade do cuidado em caso de manutenção ou retomada do uso de substâncias psicoativas. Nesse sentido, os resultados obtidos conforme as afirmações foram: "Acho correto continuar sendo atendido mesmo após voltar a fazer uso de álcool ou drogas" ( $\mathrm{M}=4,74$. $\mathrm{DP}=0,85)$; "Reorganizar o plano terapêutico após voltar a fazer uso de álcool ou drogas dá mais coragem para continuar o acompanhamento no CAPSad" $(\mathrm{M}=4,90$. $\mathrm{DP}=0,28)$, e "No caso de voltar a fazer uso de álcool ou drogas, eu voltaria ao CAPSad" $(\mathrm{M}=4,88$. $\mathrm{DP}=0,34)$.

No que se refere às pessoas importantes para os usuários do serviço, foi possível constatar que familiares e companheiro(a) exercem maior influência sobre os aspectos que envolvem a utilização de medicamentos, do que outras pessoas que não fazem parte da família $[F(2,21)=6,88 ; \mathrm{p}<0,01]$.
Com relação às atitudes dos usuários frente às práticas de cuidado, as questões envolveram as esferas cognitivas, comportamentais e afetivas das atitudes. Assim, com relação aos elementos acolhimento e profissional de referência os participantes apresentaram uma atitude predominantemente favorável $(M=4,65, \mathrm{DP}=0,64)$. Em relação ao profissional de referência, os participantes se mostraram predominantemente favoráveis $(\mathrm{M}=$ $4,79, \mathrm{DP}=0,44)$.

Ao analisar isoladamente os itens que compõem as atitudes frente ao Plano Terapêutico Singular (PTS) como prática de cuidado, apesar de não haver significância estatística - considerando consenso de $95 \%$ de confiabilidade - infere-se que participar das oficinas pode estar relacionado a uma maior percepção do usuário de que o PTS está de acordo com sua necessidade $[\mathrm{t}(22)=1,79 ; \mathrm{p}=$ $0,087]$. Ainda, constata-se que quem participa das oficinas terapêuticas refere gostar de realizar as atividades de grupo $[\mathrm{t}(22)=2,94 ; \mathrm{p}$ $<0,01]$.

Em relação às oficinas terapêuticas obteve-se uma atitude favorável $(\mathrm{M}=4,42$ $\mathrm{DP}=0,85)$ por parte dos participantes. Quanto às assembleias de usuários, os participantes se mostraram predominantemente favoráveis $(\mathrm{M}=$ 4,04 $\mathrm{DP}=0,97)$. Ressalta-se que apesar de serem favoráveis, dos 24 participantes, 10 não participam das assembleias, o que demonstra uma dissociação entre a atitude e o comportamento efetivamente realizado. Notadamente, ao analisar apenas a atitude dos usuários que participam das assembleias ( $\mathrm{n}=$ 14) se observa uma redução do nível de favorabilidade $(M=3,79 ; D P=1,32)$. Assim, essa prática, apesar de ser uma importante ferramenta de efetivação da autonomia do usuário, não reflete uma ação constituída com ampla efetividade pelos mesmos, visto que a favorabilidade reduz.

Ao se analisar os dados obtidos no cruzamento das atitudes frente à assembleia de usuários com as variáveis "tempo de frequência no CAPSad" e "participação em oficinas terapêuticas" percebe-se que os usuários que 
frequentam o CAPSad a menos tempo, apesar de gostarem mais de participar das assembleias em comparação aos usuários que frequentam a mais tempo, são menos favoráveis a emitir suas opiniões nas assembleias. Essa diferença aparece principalmente entre os usuários com mais de 3 anos de participação em comparação com os de 3 a 6 meses $[\mathrm{f}(2,22)=0,6032 ; \mathrm{p}<$ $0,05]$ e de 6 meses a 1 ano $[F(2,21)=0,584 ; p$ $<0,05]$.

$\mathrm{O}$ atendimento individual como prática de atenção à saúde obteve dos participantes um posicionamento favorável $(\mathrm{M}=4,66 ; \mathrm{DP}=$ 0,85). Em relação à prática de visitas domiciliares, os participantes também manifestam atitude favorável $(\mathrm{M}=4,50 ; \mathrm{DP}=$ $0,91)$, bem como a prática de medicação $(\mathrm{M}=$ $4,63, \mathrm{DP}=0,64)$.

\section{Discussão}

Em relação à caracterização dos participantes, a prevalência do sexo masculino em serviços como o CAPSad também foi confirmada em outros estudos (Faria \& Schneider, 2009; Mangualde et al., 2013; Borges, 2016; Oliveira et al., 2017; França, 2018). Observa-se que a população masculina é a mais atingida pelo uso prejudicial de drogas, gerando uma procura maior pelo cuidado nos serviços de saúde, em comparação ao sexo feminino. Além disso, questões culturais, fundamentadas em estereótipos e preconceitos relacionados aos papéis de gênero na sociedade exercem importante influência sobre esse fenômeno (Faria \& Schneider, 2009).

Além disso, a procura pelo atendimento nos serviços públicos como o CAPSad é mais frequente na população de menor nível socioeconômico, variável esta que é historicamente relacionada à escolaridade. Ao analisar a baixa escolaridade do indivíduo e de seus familiares, associada à renda familiar insuficiente, reflete-se sobre a relação destas com o uso prejudicial de drogas, visto que uma ampla parcela da sociedade vive constantemente ameaçada pela instabilidade nas condições de vida e pela exclusão social (Veiga et al., 2016; Oliveira et al., 2017), situação que amplia os fatores de risco e que, por sua vez, potencializam a vulnerabilidade social.

O contexto familiar representa um importante papel na relação do usuário com o fenômeno das drogas. A família enquanto contexto de influência comportamental pode representar um duplo papel. Pode ser um fator de risco para o uso de substâncias, na medida em que seja um ambiente de exposição ao uso, além de antecedentes familiares de violência doméstica e pressão do grupo que podem contribuir para desfechos negativos à saúde (Borges, OMoré, Krenkel, \& Schneider, 2017; França, 2018). Bem como, um fator de proteção ao uso, quando há vínculos que possibilitam a comunicação de um conjunto de normas e sistemas de crenças que orientam expectativas sobre os papéis sociais e sobre o modo de vida. Assim, a rede familiar pode ser considerada tanto um fator de proteção quanto de risco (Borges et al., 2017), sendo necessário assim ampliar o foco de atuação das equipes de modo a abranger o cuidado também aos familiares.

Se destaca nessa pesquisa o acesso dos usuários ao serviço por demanda espontânea, tendo em vista que 15 participantes acessaram diretamente o CAPSad por vontade própria ou por indicação de pessoas próximas. Essa mesma característica foi encontrada em estudo de Barbosa (2017). A demanda espontânea é facilitada ao CAPSad por este se tratar de um serviço de porta aberta à comunidade, sem obrigatoriedade de um encaminhamento de outro serviço de saúde.

Outro dado importante se refere ao profissional de referência presente no serviço. Segundo Grigolo, Peres, Garcia Junior, \& Rodrigues (2015), a vinculação terapêutica entre o profissional de referência e o usuário estimula a produção de um novo padrão de responsabilidade pela coprodução de saúde entre estes dois agentes. Diminui a distância entre este profissional e o usuário, deixa-se de objetivar a doença do sujeito para destacar a pessoa e toda sua complexidade. Sendo o momento de acolhimento essencial para a construção desse vínculo. O profissional de 
referência também possui papel importante em atendimentos pontuais, atuando de forma a estimular a autonomia dos usuários (Oliveira, Nunes, \& Nunes, 2019).

Quanto ao consumo ou não de drogas, observa-se visões antagônicas entre os participantes. Há, portanto, atitudes tanto favoráveis quanto desfavoráveis à frequentar o CAPSad enquanto se faz uso de substâncias. No Brasil, há diferentes metodologias de cuidado à questão do uso de drogas e no contexto atual, as duas perspectivas que mais se destacam são o Alcoólicos Anônimos (AA) e a de atenção psicossocial do CAPSad. Integrantes dos AA seguem o princípio da abstinência total, orientados pela metodologia dos 12 passos de Bill e Bob, enquanto o CAPSad segue o princípio da redução danos, proporcionando ao usuário ações preventivas e oferecendo cuidados nos aspectos biopsicossociais (Carvalho, Liotti, \& Lenzi, 2015).

Assim, a política de redução de danos no contexto do uso de drogas é uma estratégia que pode ser utilizada quando já se faz consumo da substância ou para evitar o consumo da mesma, tendo como objetivo minimizar os riscos causados pelo uso da droga. A política de Redução de Danos preconiza que a abstinência não seja o único objetivo a ser alcançado, pois quando se trata de vidas humanas se preconiza a singularidade e as possíveis escolhas feitas pelos sujeitos (Ministério da Saúde, 2003).

Nesse sentido, deve-se acolher cada usuário conforme suas demandas e com o que é possível ofertar, com o objetivo de estimular a participação e engajamento no serviço. Portanto, as estratégias de redução de danos buscam melhorias na qualidade de vida, evitando a marginalização por meio da inserção na rede de saúde e assistência social (Gomes \& Vecchia, 2018). No entanto, a Nota Técnica $n^{\circ}$ $11 / 2019$, que propõe mudanças na Política Nacional de Saúde Mental e nas Diretrizes da Política Nacional sobre Drogas, facilita a internação dos sujeitos em comunidades terapêuticas, não tendo mais como base a redução de danos (Decreto n. 9.761, 2019).
No que se refere ao componente de crenças presente na atitude dos participantes, foi lançado foco especialmente sobre a lógica de redução de danos e sobre o cuidado quando há manutenção do uso de substâncias pelos usuários. Tendo em vista que as crenças são componentes intrínsecos às atitudes, elas possuem papel de influência sobre a intenção dos indivíduos em realizar comportamentos específicos ou engajar-se em práticas sociais (Ajzen, 2005; Moutinho \& Roazzi, 2010).

Assim, ao analisar os escores gerais verifica-se que há um posicionamento sustentado por crenças predominantemente favoráveis aos aspectos ligados à redução de danos. Estes dados são explicados ao se observar que os usuários que buscam o serviço e que já frequentam o mesmo, fazem uso de substância e estão no CAPSad em busca de uma melhora na saúde e diminuição do uso ou cessação do mesmo, sendo que 11 dos 24 participantes referiram ter procurado o CAPSad para reduzir ou interromper o uso. Além disso, existe um processo de influência mútua entre comportamento e posicionamento atitudinal frente a determinados objetos sociais, buscando ao máximo a redução de uma dissonância entre eles (Tróccoli, 2011). Tais reflexões vão ao encontro da lógica da redução de danos, que não tem como objetivo primário a cessação do uso, e sim, por meio de um conjunto de medidas visa minimizar as consequências do uso de substâncias psicoativas e o fortalecimento dos fatores de proteção (Ministério da Saúde, 2015a; 2015b).

Ao focar-se em práticas que são alvo de comportamentos, como por exemplo, práticas de cuidado em saúde, é necessário abordar as normas subjetivas, que consistem na influência do meio, em forma de pressão social para que a pessoa realize ou não determinado comportamento, sobretudo a pressão exercida por pessoas consideradas importantes para o indivíduo (Ajzen, 2005; Moutinho \& Roazzi, 2010). As normas sociais, da mesma forma que as crenças, fazem parte do contexto de atitude dos indivíduos frente aos objetos sociais. Essa relação se processa em um contínuo de influências mútuas entre esses elementos. 
Assim, ao verificar a influência das pessoas consideradas mais importantes para os participantes, principalmente familiares e companheiros(as).

Quanto à prática de acolhimento realizada pelos profissionais, os dados demonstram que é um importante dispositivo para a adesão aos cuidados propostos pela equipe ao seu PTS (Fonseca, Pereira, Alencar, Lage, \& Silveira, 2014; Lima, Rodrigues Neto, Coelho, Marques, \& Lotif, 2015). Através desta prática o participante desenvolve um vínculo maior com este profissional, pois estabelece maior contato através de atendimentos individuais e diálogos informais, bem como, através da (re)elaboração do PTS, a fim de potencializar sua ação terapêutica. Levando em conta que o usuário possui uma participação ativa em sua elaboração, planejado de acordo com as necessidades referidas por ele mesmo e revisto quando necessário (Ministério da Saúde, 2015a; 2015b). Tendo em vista um posicionamento favorável a essa prática, é possível que o comportamento dos participantes em relação ao PTS demonstre um compromisso maior com seu próprio cuidado.

A participação dos usuários em diversos espaços dos CAPS também deve ser levada em consideração. Nas oficinas terapêuticas os usuários dos CAPSad têm uma participação ativa quanto a sugestões de temas a serem abordados ou a adesão nas oficinas que lhes despertam interesse (Ministério da Saúde, 2015b). Enquanto nas assembleias de usuários se faz uma discussão sobre os atendimentos e serviços prestados pelo CAPSad, em que os usuários podem expressar sua opinião, bem como, avaliar e propor mudanças para melhorias no serviço (Ministério da Saúde, 2015a, 2015b).

No entanto, verificou-se uma dissociação entre a atitude e o comportamento efetivo, pois por mais que sejam favoráveis as assembleias, não participam. Ajzen (2005) explica que a relação entre o complexo atitudes, normas e crenças - que resulta em uma intenção comportamental - com a realização do comportamento não se dá em um processo exatamente estável. A correlação entre a intenção em se comportar e a realização do comportamento é situacional, havendo nesse sistema variáveis ambientais que impedem a efetiva realização do comportamento.

Lançando essa teorização sobre o contexto da atitude dos participantes frente à assembleia de usuários, reflete-se que é primordial buscar a ampliação das facilitações do ambiente para aumentar as chances de os usuários participarem das assembleias, tendo em vista sua atitude favorável. Como consequência, há risco de perda de potencialidade do cuidado, pois o espaço da assembleia de usuários permite uma participação ativa por meio de ações que influenciam a rotina e a qualificação das práticas ofertadas no serviço de saúde (Silva \& Wojcikowski, 2011). Verificou-se também que quanto maior o tempo no serviço, maior a favorabilidade em frequentar as assembleias.

Nesse sentido, para existir um sentimento de pertencimento a um determinado grupo é preciso que os membros se identifiquem como pertencentes a ele. Há também uma diferenciação cognitiva, afetiva e comportamental dos grupos, caracterizando as interações que ocorrem no endogrupo e no exogrupo (Alfinito \& Corradi, 2011). A distinção entre endogrupo e exogrupo pode ser feita através de duas categorias respectivamente, os usuários antigos ou de mais tempo (endogrupo - senso de pertencimento) e os usuários que frequentam o serviço a menos tempo (exogrupo).

Reflete-se que uma vez que os usuários se apropriam do funcionamento do CAPSad através da interação com a equipe e com as atividades propostas, é possível um maior engajamento nas discussões que acontecem nas assembleias de usuários. Ainda, através das suas participações desenvolvem a capacidade de identificar as necessidades e possíveis falhas do funcionamento do serviço, sendo a assembleia uma abertura para discussão de críticas, sugestões e possíveis melhorias (Constantinidis, Cid, Santana, \& Renó, 2018). 
Quanto aos demais espaços da instituição, surge a favorabilidade sobre os atendimentos individuais, visitas domiciliares e uso de medicação. Pacheco, Rodrigues e Benatto (2018) defendem que o espaço do atendimento individual propicia um contato mais direto e preciso entre profissional e usuário, o que possibilita um vínculo maior para a abordagem de temas que se referem à vida particular e à intimidade. Esse elemento pode estar ligado à favorabilidade dos usuários com essa modalidade de prática de atendimento, uma vez que o usuário passa a ter um espaço para se expressar de forma mais sigilosa e direta com o profissional que está lhe atendendo.

Por outro lado, o atendimento individual apresenta um importante desafio: propiciar o movimento da atenção especializada aos usuários de saúde mental historicamente focada em atendimentos individualizados e de caráter ambulatorial para a perspectiva de atenção integral e clínica ampliada, atingindo ativamente as demais esferas da vida do sujeito. A partir disso, defende-se a variedade de ações que os profissionais podem realizar, como oficinas terapêuticas, grupos operativos e visitas domiciliares, como ações que diversificam as modalidades de atendimento e contribuem para o aumento da responsabilidade do usuário com sua saúde (Duarte, Viana, \& Olschowsky, 2016; Pereira \& Palma, 2018).

Enquanto a visita domiciliar é um importante suporte para o atendimento aos usuários, que não ficam desamparados quando há esta necessidade. Está prática promove o estabelecimento e fortalecimento do vínculo entre usuário e equipe, além de ser um momento importante para a equipe visualizar o contexto social em que o usuário se encontra, e por isso, uma ferramenta para planejar uma assistência qualificada que contemplem as necessidades do indivíduo (Vargas et al., 2016).

Quanto ao uso de ansiolíticos e antipsicóticos, Carvalho, Liotti e Lenzi (2015) e Fonseca, Gondim e Fonteles (2014), explicam que em muitos casos existe uma crença de que a medicação por si mesma seria suficiente para a resolução do uso das drogas. Motivada por efeitos como a redução dos níveis de ansiedade, auxílio ao processo de desintoxicação e controle dos efeitos da cessação do uso. Apesar disso, ressalta-se a importância de lançar o olhar sobre as práticas de grupo e atividades terapêuticas coletivas como ferramentas de desmedicalização do usuário de saúde mental.

\section{Considerações finais}

Com base nas teorizações da Psicologia Social aplicadas ao campo da Saúde Mental, especificamente à questão que envolve a atenção aos usuários de álcool e outras drogas, lançou-se investigação sobre as atitudes frente às práticas de cuidado no CAPSad, na busca de compreensão sobre a participação do usuário no serviço de saúde e em seu próprio cuidado. Esse cenário se conecta a aspectos históricos, socioculturais, econômicos, políticos e individuais, afinal, sabe-se que o uso de drogas está presente em diferentes contextos, com objetivos e motivações diversas.

Assim, quanto ao posicionamento atitudinal foi possível constatar que a maioria dos participantes apresentou uma atitude positiva em relação às práticas de cuidado que o CAPSad oferece. Entende-se que a favorabilidade dos participantes oportuniza vínculo positivo com a equipe profissional e com o serviço de saúde, potencializando as práticas, a frequência na instituição e adesão às propostas de seu Projeto Terapêutico Singular.

Se destaca a questão das assembleias de usuários e os atendimentos individuais. Há um grande número de usuários que não participam das assembleias, enquanto a maioria dos participantes preferem o atendimento individual. Neste sentido, defende-se que a participação nas assembleias deve ser incentivada, sobretudo quando se relaciona à autonomia do usuário, que deve ser foco de ação do CAPSad. Bem como, ao controle social proposto como um dos pilares do SUS, por meio da gestão participativa (gestores, profissionais e usuários). 
$\mathrm{O}$ atendimento individual é uma prática não prioritária do serviço, mas que para o usuário demonstra a segurança do "confidencial" e privado, que também se faz necessário. Sendo assim apresentado o desafio de tornar as intervenções coletivas e grupais um ambiente seguro e acolhedor como o atendimento individual. Observou-se também que os participantes que frequentam as oficinas terapêuticas apresentam maior participação nas assembleias. Considerando-se que as oficinas são espaços que estimulam a elaboração dos conflitos internos e externos, pode-se inferir que estes se apropriam mais da vivência no serviço, interagem com os demais usuários, identificam a pertença ao grupo e buscam maior protagonismo em sua situação de saúde.
Salienta-se que o estudo apresenta limitações relacionadas à quantidade de participantes entrevistados. Assim, por tratar-se de uma amostra pequena, o poder de explicação dos dados tem sua margem reduzida, gerando necessidades de ampliação das estratégias de investigação em estudos futuros. Em complemento, é sugerido que futuras pesquisas que abordem essa temática ampliem a exploração dos aspectos relacionados às crenças e normas sociais, associando instrumentos quantitativos e qualitativos que permitam captar o conteúdo intrínseco desses fenômenos, tão ricos para o campo teórico e prático da psicologia social e da saúde.

\section{Referências}

Ajzen, I. (2005). Attitudes, personality and behavior. New York: Open University Press.

Alfinito, S., \& Corradi, A. A. (2011). Contato Intergrupal: Conflito Realístico, Privação Relativa e Equidade. In C. V. Torres, \& E. R. Neiva (Org.), Psicologia social: principais temas e vertentes (pp. 262-286). Porto Alegre: Artmed.

Barbosa, J. S. P. (2017). O perfil dos idosos usuários de drogas atendidos nos Centros de atenção psicossocial álcool e drogas no Distrito Federal (Dissertação de mestrado). Universidade de Brasília - UNB, Brasília, DF, Brasil.

Borges, C. D. (2016). Relação entre rede social significativa e atenção à saúde de usuários de CAPSad com problemas relacionados ao uso de álcool (Dissertação de mestrado). Universidade Federal de Santa Catarina - UFSC, Florianópolis, SC, Brasil.

Borges, C. D., OMoré, C. L. O. O., Krenkel, S., Schneider, D. R. (2017). Família, redes sociais e o uso de drogas: tensionamento entre o risco e a proteção. Pesquisas e Práticas Sociais, 12(2), 405-421. Recuperado de http://seer.ufsj.edu.br/index.php/revista_pp p/article/view/2588

Carlini, E. L. A, Noto, A. R., Sanchez, Z. M., Carlini, C. M. A., Locatelli, D. P., Abeid, L. R... Moura, Y. G. (2010). VI

Levantamento nacional sobre o consumo de drogas psicotrópicas entre estudantes do ensino fundamental e médio das redes pública e privada de ensino de 27 capitais brasileiras. São Paulo: CEBRID - Centro Brasileiro de Informações sobre Drogas Psicotrópicas, UNIFESP - Universidade Federal de São Paulo.

Carvalho, J. E. S., Liotti, D. B. M., \& Lenzi, M. C. R. (2015). CAPSad e Alcoólicos Anônimos: O processo de tratamento sobre o ponto e vista dos usuários. Cadernos Brasileiros de Saúde Mental, 7(16), 41-61. Recuperado de http://stat.entrever.incubadora.ufsc.br/index .php/cbsm/article/view/2339

Constantinidis, T. C., Cid, M. F. B., Santana, L. M., \& Renó, S. R. (2018). Concepções de Profissionais de Saúde Mental acerca de Atividades Terapêuticas em CAPS. Trends Psychology, 26(2), 911-926. doi: 10.9788/TP2018.2-14Pt

Decreto $n^{\circ}$ 9.761, de 11 de abril de 2019. Aprova a Política Nacional sobre Drogas. 
Recuperado de

http://www.in.gov.br/materia/-

/asset_publisher/Kujrw0TZC2Mb/content/i d/71137357/do1e-2019-04-11-decreto-n-9-

761-de-11-de-abril-de-2019-71137316

Doise, W. (2001). Atitudes e representações

sociais. In D. Jodelet (Org.), As

representações sociais (pp. 187-203). Rio

de Janeiro: EdUERJ.

Duarte, M. L. C., Viana, K., \& Olschowsky, A. (2016). Atenção a pessoas dependentes de crack em um centro de atenção psicossocial. Revista Contexto \& Saúde, 16(31), 165-172. doi: $10.21527 / 2176-$ 7114.2016.31.165-172

Faria, J. G., \& Schneider, D. R. (2009). O perfil dos usuários do Capsad-Blumenau e as políticas públicas em saúde mental. Psicologia \& Sociedade, 21(3), 324-333. doi: 10.1590/S0102-71822009000300005

Ferreira, P. S., \& Luiz, M. A. V. (2004). Percebendo as facilidades e dificuldades na implantação de serviços abertos em álcool e drogas. Texto \& Contexto Enfermagem, 13(2), 209-216. Recuperado de https://www.redalyc.org/pdf/714/71413203. pdf

Fonseca, N. F., Gondim, A. P., \& Fonteles, M. M. F. (2014). Influência dos grupos terapêuticos em Centro de Atenção Psicossocial entre usuários com dependência de cocaína/crack. Saúde debate, 28(102), 551-561. doi: 10.5935/0103-1104.20140051

Fonseca, R. L., Pereira, C. V., Alencar, C. A., Lage, M. A. M. O., \& Silveira, A. R. (2014). Percepção de usuários de crack sobre o tratamento em um Centro de Atenção Psicossocial Álcool e outras Drogas. Revista de Atenção Primária à Saúde, 17(2), 214-222. Recuperado de https://periodicos.ufjf.br/index.php/aps/artic le/view/15231

França, A. C. S. (2018). Perfil dos usuários do centro de atenção psicossocial álcool e outras drogas (CAPS-AD III) no município de Limoeiro-PE (Trabalho de conclusão de curso). Universidade Federal de Pernambuco - UFPB, Vitória de Santo Antão, PE, Brasil.
Gomes, T. B., \& Vecchia, M. D. (2018).

Estratégias de redução de danos no uso prejudicial de álcool e outras drogas: revisão de literatura. Ciência e Saúde Coletiva, 23(7), 327-2327. doi: 10.1590/1413-81232018237.21152016

Gonçalves, S. S. P. M., \& Tavares, C. M. M. (2007). Atuação do Enfermeiro na Atenção ao Usuário de Álcool e Outras Drogas nos Serviços Extra-Hospitalares. Escola Anna Nery Revista de Enfermagem, 11(4), 586592. doi: 10.1590/S141481452007000400005

Grigolo, T. M., Peres, G. M., Garcia Junior, C. A., \& Rodrigues, J. (2015). O projeto terapêutico singular na clínica da atenção psicossocial. Cadernos Brasileiros de Saúde Mental, 7(15), 53-73. Recuperado de http://stat.necat.incubadora.ufsc.br/index.ph $\mathrm{p} / \mathrm{cbsm} /$ article/view/2951

Lima, M. Z., Rodrigues Neto, E. M., Coelho, M. O., Marques, L. A. R. V., \& Lotif, M. A. L. (2015). Percepção do cuidado em saúde no capsad: uma visão do paciente. Saúde (Santa Maria), 41(1), 239-248. doi: $\underline{10.5902 / 2236583415619}$

Lima, M. L. \& Correia, I. (2013). Atitudes: medida, estrutura e funções. In J. Vala, \& M. B. Monteiro (Orgs.), Psicologia Social (pp. 201-243). Lisboa: Fundação Calouste Gulbeinkian.

Mangualde, A. A. S., Ananias A., Botelho, C. C., Soares, M. R., Costa, J. F., Junqueira, A. C. M... Vidal, C. E. L. (2013). Perfil epidemiológico dos pacientes atendidos em um Centro de Atenção Psicossocial. Mental, 10(19), 235-248. Recuperado de https://www.redalyc.org/pdf/420/42028699 006.pdf

Ministério da Saúde. (2003). A Política do Ministério da Saúde para atenção integral a usuários de álcool e outras drogas. Brasília: Ministério da Saúde.

Ministério da Saúde. (2015a). Centros de Atenção Psicossocial e Unidades de Acolhimento como lugares de atenção psicossocial nos territórios: orientações para elaboração de projetos de construção, reforma e ampliação de CAPS e de UA. Brasília: Ministério da Saúde. 
Ministério da Saúde. (2015b). Cadernos HumanizaSUS: Saúde Mental (Vol. 5). Brasília, DF: Ministério da Saúde.

Moutinho, K., \& Roazzi, A. (2010). As teorias da Ação Racional e da Ação Planejadas:

Relações Entre Intenções e Comportamentos. Avaliação Psicológica, 9(2), 279-287. Recuperado de https://www.redalyc.org/pdf/3350/3350272 83012.pdf

Oliveira, M. V. P., Nunes, E. P. O., \& Nunes, J. R. (2019). Reinserção profissional da mulher em tratamento no CAPS AD III em Palmas-TO. Revista Humanidades e Inovação, 6(12), 250-261. Recuperado de https://revista.unitins.br/index.php/humanid adeseinovacao/article/view/1242

Oliveira, V. A., Capistrano, F. C., Ferreira, A. C. Z., Kalinke, L. P., Felix, J. V. C., \& Maftum, M. A. (2017). Perfil sociodemográfico e clínico de pessoas atendidas em um CAPS ad do sul do Brasil. Revista Baiana de Enfermagem, 31(1), e16350. Recuperado de https://periodicos.ufba.br/index.php/enferm agem/article/view/16350/14059

Pacheco, S. U. C., Rodrigues, S. R., \& Benatto, M. C. (2018). A importância do empoderamento do usuário de CAPS para a (re)construção do seu projeto de vida. Mental, 12(22), 72-89. Recuperado de http://pepsic.bvsalud.org/scielo.php?script= sci_arttext\&pid=S167944272018000100006

Pereira, O. P., \& Palma, A. C. R. (2018). Sentidos das oficinas terapêuticas ocupacionais do CAPS no cotidiano dos usuários: uma descrição fenomenológica. Revista da Abordagem Gestáltica, 24(1), 15-2. doi:10.18065/RAG.2018v24n1.2

Portaria GM n. 336, de 19 de fevereiro de 2002. Define e estabelece diretrizes para o funcionamento dos Centros de Atenção Psicossocial. Recuperado de http://bvsms.saude.gov.br/bvs/saudelegis/g m/2002/prt0336_19_02_2002.html

Silva, J. P., \& Wojcikowski, M. A. P. (2011). Representações Sociais na Atenção Básica à Saúde: investigação sobre a corresponsabilidade dos usuários na Estratégia Saúde da Família (ESF) (Trabalho de conclusão de curso). Curso de Psicologia - Faculdade Metropolitana de Guaramirim, Guaramirim, SC, Brasil.

Tróccoli, B. (2011). Cognição social. In: C. V. Torres, \& E. R. Neiva (Orgs.), Psicologia social: principais temas e vertentes (pp. 7999). Porto Alegre: Artmed.

Vargas, C. B. V. et al. (2016). A visita domiciliar como estratégia de acolhimento a famílias de dependentes químicos. Revista Latinoamericana Ambiente e Saúde, 4(1).

Veiga, L. D. B., Santos, V. C., Santos, M. G., Ribeiro, J. F., Amaral, A. S. N., Nery, A. A... Casotti, C. A. (2016). Prevalência e fatores associados à experimentação e ao consumo de bebidas alcoólicas entre adolescentes escolares. Cadernos de Saúde Coletiva, 24(3), 368-375. doi: $\underline{10.1590 / 1414-462 X 201600030037}$ 


\section{Dados sobre os autores:}

- Débora Mariana Stahelin: Possui graduação em Psicologia pelo Centro Universitário Leonardo da Vinci (2016), Especialização em Terapia Cognitivo Comportamental (2019), Especialização em Terapia Familiar (2021) e Especialização em Terapia de Casal (2021) pela UNIBF. Atualmente é psicóloga atuando na Clínica Corpo e Mente.

- Jairson José Leichtweis Reis: Possui graduação em Psicologia pela Sociedade Educacional do Vale do Itapocu (2016), Especialização em Acupuntura pela Logos University (2020). Atualmente é Acupunturista da Clínica Corpo e Mente.

- Jean Paulo da Silva: Doutorando do Programa de Pós-graduação em Psicologia da Universidade Federal de Santa Catarina (UFSC) na área de Psicologia Social e Cultura, linha de pesquisa em Representações e Práticas sociais. Mestre em Psicologia pela mesma instituição na área de Processos Psicossociais, Saúde e Desenvolvimento Psicológico. Graduado em Psicologia pela Faculdade Metropolitana de Guaramirim (FAMEG). Docente do curso de Psicologia da UNIVINCI (Guaramirim - SC). Atua também na Secretaria da Saúde de Massaranduba - SC como Coordenador de Planejamento e Educação Permanente em Saúde.

- Marieli Mezari Vitali: Possui graduação em Psicologia pela Universidade do Extremo Sul Catarinense (2018). Especialista em Psicodrama no Centro Universitário Amparense (UNIFIA) e Psicodramatista Nível I pela Escola Viver Mais Psicologia (2020). Mestre em Psicologia pela Universidade Federal de Santa Catarina - UFSC. Atualmente é doutoranda em Psicologia no Programa de Pós-Graduação em Psicologia, da Universidade Federal de Santa Catarina - UFSC, com ênfase em Psicologia Social; e Formação em Psicodrama Nível Didata em andamento pela Escola Viver Mais Psicologia. Tem experiência na área de Psicologia, com ênfase em Psicologia Social, atuando principalmente nos seguintes temas: psicologia social, representações sociais, saúde mental e psicodrama.

A submissão de originais para este periódico implica na transferência, pelos autores, dos direitos de publicação impressa e digital. Os direitos autorais para os artigos publicados são do autor, com direitos do periódico sobre a primeira publicação. Os autores somente poderão utilizar os mesmos resultados em outras publicações indicando claramente este periódico como o meio da publicação original. Em virtude de sermos um periódico de acesso aberto, permite-se o uso gratuito dos artigos em aplicações educacionais e científicas desde que citada a fonte conforme a licença CC-BY da Creative Commons. 